\title{
A New Interpolation-Based Super-Resolution Algorithm by the Cubic Spline Interpolation for Edge Pixels and Iterative Update Method
}

\author{
Chun-Jiang Duanmu, Di Zhao \\ Dept. Of Information Engineering, College of Mathematics, Physics, and Information Engineering, \\ Zhejiang Normal University, \\ Jin Hua city, P. R. China \\ E-mail: duanmu@zjnu.cn
}

\begin{abstract}
Since the interpolation-based image super-resolution algorithms can be executed very fast, it is the research focus of this paper. However, in the traditional interpolation-based super-resolution algorithms, the image edges are usually blurred and the quality of the reconstructed image is not satisfied. For this reason, this paper proposes a new interpolation algorithm. It first extracts the edge pixels by using the Prewitt operator. Then, the edge pixels are interpolated along the edge direction by cubic spline interpolation, so that there is little image edge blurs. After this, a recursive update process is applied to the high-resolution image to boost the system performance, until the degraded high-resolution image is very similar to the low-resolution image. Simulation results show that the proposed algorithm performs much better than the traditional interpolation-based algorithms.
\end{abstract}

Keywords-image processing; super-resolution; interpolationbased super-resolution

\section{INTRODUCTION}

With the rapid development of the digital camera, digital video, digital television, etc. and the internet technique, the people's need for the high resolution digital images are increasing significantly. However, with the bandwidth limit of the transmission network and the storage space limit of the network nodes, the costs for the storage and the transmission of high-resolution images are huge and sometimes are unacceptable and unimplementable. For this reason, the image compression technique has been proposed to partially solve the problem. The international image and video standards such as the JPEG standard, the JPEG 2000 standard, the MPEG series standards, and the H.26x series standards, have been put forward to reduce the output bits to represent the images or videos at the source transmission end using data compression technique. Then, these bits are transmitted through the network to the end point are forwarded to the decompression tools to render the reconstructed images or videos. However, due to the unstable conditions of the network, the compressed bits transferring in the network are very prone to various kinds of interference and network jams. When the network jams happen, the usual solution is to drop the packets in the network. Thus, there usually a lot of the bit errors for the transferred compressed bits at the end point. Since the compressed bits are very sensitive to bit errors, a single bit of error usually causes a lot of the following bits to be incorrectly decoded until a new synchronization point in the bit stream. Furthermore, due to the compression, a lot of reconstruction artifacts, such as the block artifact, the ringing artifact, and degradation of the images occur even without the transmission errors. Thus, the quality of the images at the end point usually cannot meet the human needs. To solve this problem, recently the single frame super-resolution technique has been put forward to increase the resolution of an image frame. The objective of this technique is to construct an image of higher resolution than the original input image with relatively good quality. In this way, this technique can be combined with the data compression methods mentioned previously to transmit or store low resolution images at the source end and only transfer the compressed bits of low-resolution images. Then, at the receiver end, the images are firstly decoded and then put as the source for super-resolution process. Since only the bits to represent the low-resolution iimages are transferred, a lot of number of bits can be saved compared with the original scheme. Thus, the number of bits to be transferred are save dramatically. Moreover, the space to store the low-resolution images are much less than that to store the high-resolution images, so that a lot of space to store the images on the network can be saved. Although only the low-resolution images are obtained at the receiver end, the quality of the reconstructed images by superresolution is usually acceptable and pleasing to human eyes. Due to these reasons, the research on single frame superresolution technique has been surged recently.

In the current research on the single frame superresolution, the algorithms in the literature can be classified over three main approach. In the first approach, the lowresolution images are interpolated to get the high-resolution images. The computational complexity of these algorithms is usually very low, and thus they can be executed very fast to suit for the real-time needs. However, the quality of these algorithms are relatively low and especially the image edges of the reconstructed high-resolution images are usually blurred.

In the second approach, the low-resolution images are firstly divided into image blocks. Then, a training set consisting of the low-resolution image blocks and the corresponding high-resolution image blocks is obtained by extracting these blocks from the training high-resolution images and the corresponding degraded low-resolution blocks. After the training phase is complete, low-resolution images are put into these algorithms in the online phase to 
get the high-resolution images. In this phase, the input images are firstly divided into candidate image blocks with some overlap, and then $\mathrm{k}$ nearest neighboring image blocks in the training set which are mostly similar to the current candidate image block are selected. Then, a high resolution block is reconstructed by linearly combining the $\mathrm{k}$ highresolution image blocks corresponding to the selected $\mathrm{k}$ nearest low-resolution image blocks in the training set. These algorithms are called as the example-based algorithms, since a low-resolution image block and its corresponding high-resolution image block in the training set is called as an example. These algorithms have the following shortcomings. Firstly, the time for obtaining the training set is very long. Secondly, when the images in the training set have relatively low correlation with the candidate image to be magnified, the result of the superresolution is poor. Thirdly, the on-line phase of these algorithms are also very time-consuming, especially in the step for finding the $\mathrm{k}$ nearest neighbors.

In the third approach for obtaining the high-resolution images, the sparse representing technique is utilized. They first have to train the sparse low-resolution dictionary to represent the low-resolution image blocks, and the sparse high-resolution dictionary to represent the high-resolution image blocks from a very large number of image blocks. These training have to be coupled for the co-training of these two types of dictionaries. In the online phase of these algorithms, the candidate low-resolution image block to be magnified is firstly and optimally represented by the lowresolution sparse dictionaries in the training set. Then, a high-resolution block is obtained by using the highresolution sparse dictionaries with the representing coefficients related to the coefficients to represent the lowresolution image blocks with the low-resolution sparse dictionaries. These algorithms have the following shortcomings. Firstly, the co-training of the low-resolution and high-resolution dictionaries is very time-consuming. Secondly, similar to the example-based algorithms, when the images in the training set have low correlation with the candidate image to be magnified, the quality of the superresolution is poor. Thirdly, the online-phase of these algorithms is also very time-consuming, especially in finding the optimal coefficients for the low and high resolution image dictionaries.

From the above discussions, it can be seen that the example-based algorithms and the algorithms using the sparse technique are usually very time-consuming and cannot be used for real-time applications, especially the super-resolution video applications. It can also be seen that when the candidate low-resolution image has low correlation with the images in the training set, the reconstructed high-resolution image by using the above two classes of algorithms has a poor image quality. Furthermore, these two classes of algorithms usually have the block artifact which is unpleasant to human eyes. Due to these reasons, the focus of this paper is on the algorithms of super-resolution using interpolation. An new algorithm is proposed in this paper to boost the reconstructed highresolution image quality.
This paper has five sections. In the section 2, the exiting interpolation algorithms are briefly reviewed. Then, the proposed algorithm is described in details in the Section 3 and the corresponding experimental results are given in Section 4. The paper conclude in Section 5.

\section{REVIEW OF THE EXISTING INTERPOLATION ALGORITHMS}

\section{A. Nearest Neighbor Interpolation}

In the nearest neighbor interpolation algorithm, the unknown image value at the pixel of $y(m, n)$ is set to the image value of the pixel which is nearest to the unknown pixel among all the pixels with the known image value.

\section{B. The Bi-linear Interpolation Algorithm}

In the one-dimensional bi-linear algorithm, suppose that the image values of two pixels of are known as $x(i)$ and $x(j)$, and a new image value of $y(t)$ with the pixel lying on the line joining the above two pixels is sought after. The distance of the new pixel to the above two pixels are $\mathrm{d} 1$ and $\mathrm{d} 2$, respectively, then the value of $\mathrm{y}(\mathrm{t})$ in the bi-linear interpolation algorithm can be expressed as

$$
y(t)=\frac{d 2}{d 1+d 2} x(i)+\frac{d 1}{d 1+d 2} x(j)
$$

From this, it can be seen that if the image values do not vary linearly, the performance of this algorithm is not good. It can also be seen that if there are image edges not vertically or horizontally, the bi-linear interpolation algorithm blurs the edges, so that the image edges cannot be seen very clearly.

\section{Cubic Spline Interpolation}

In the one dimensional cubic spline interpolation, it usually needs more than two known image values of pixels. Given $\mathrm{n}+1$ image values of pixels, there are $\mathrm{n}$ intervals. In each interval of $\left[x_{i}, x_{i+1}\right]$, the spline function $S(x)$ has the following properties. (1) $S(x)=S\left(x_{i}\right)$, (2) the derivatives of order one and order two of the $S(x)$ are continuously. Thus, the spline function inside the $\left[x_{i}, x_{i+1}\right]$ can be expressed as

$$
S(x)=a_{i}+b_{i}\left(x-x_{i}\right)+c_{i}\left(x-x_{i}\right)^{2}+d_{i}\left(x-x_{i}\right)^{3}
$$

where the $a_{i}, b_{i}, c_{i}, d_{i}$ are the coefficients to be solved using the above two properties. From above, it can be seen that the spline function is more smooth than the linear interpolation function. Thus, usually it performs better than the linear interpolation. 


\section{Bi-cubic Interpolation}

In the bi-cubic spline interpolation, it uses the 16 pixels with known image values around the current pixel to estimate the current unknown image value. The Matlab software can implement the bi-cubic interpolation in a single function call. It usually performs better than the previous three algorithms. However, this algorithm can also blur the image edges.

\section{THE PROPOSED ALGORITHM}

In the proposed algorithm, the image edges are firstly detected via the Prewitt gradient operator. For the normal image pixels which do not lie on an image edge, the proposed anisotropic filtering is applied to the magnified pixels using the bi-cubic interpolation. Then the center edge pixel of a $5 \times 5$ window is first processed by the edge direction quantization, and then the cubic spline interpolation is carried out on the edge line to determine the image value of the center edge pixel. After all the pixels are up-scaled, a degradation process is carried for the currently reconstructed high resolution image to simulated the degradation of a high resolution image to the low resolution image. Then, the pixels whose difference value between the original image value and the degraded image value are larger than a threshold are selected. The image values of the high resolution pixel which is around a selected pixel are recomputed using a different anisotropic operator. When all the pixels around the selected pixel are processed, another iteration of the degradation and recomputed process is carried out until there are none selected pixels in the simulated low-resolution image. At this point, the reconstructed high-resolution image is the result of the proposed algorithm.

\section{A. The Detection of the Image Edge Pixels}

The Prewitt operator is convoluted with each lowresolution image pixel, i.e. at each low-resolution pixel location, the following two operators of $G_{x}$ and $G_{y}$ are convoluted with the image values, and the image gradient magnitude value is $G_{m}$, and the edge direction angle is $\theta_{m}$

$$
\begin{gathered}
G_{x}=\left[\begin{array}{lll}
-1 & 0 & 1 \\
-1 & 0 & 1 \\
-1 & 0 & 1
\end{array}\right] \\
G_{y}=\left[\begin{array}{ccc}
-1 & -1 & -1 \\
0 & 0 & 0 \\
1 & 1 & 1
\end{array}\right] \\
G_{m}=\sqrt{G_{x}^{2}+G_{y}^{2}}
\end{gathered}
$$

$$
\theta_{m}=\arctan \left(\frac{G_{y}}{G_{x}}\right)
$$

In the proposed algorithm, only the pixel whose $G_{m}>T_{1}$ is set as the edge pixel.

\section{B. Process for the Smooth Image Pixels}

The non-edge pixels in the image are smooth pixels. For the smooth pixels, they are firstly bi-cubic interpolated. Then, anisotropic operations are carried out for these pixels to more accurately estimate these pixel values. The anisotropic operation can be expressed as

$$
f(i, j, t+1)=f(i, j, t)+\beta^{*} \operatorname{div}(\alpha \times m(|\nabla f|))
$$

Where $f(i, j, t)$ and $f(i, j, t+1)$ are the image values at the $\mathrm{t}$-th iteration and $(\mathrm{t}+1)$-th iteration, respectively, $|\nabla f|$ is the magnitude of the gradient of $f(i, j, t), m(x)$ is a monotonic decreasing function, $\operatorname{div}$ is the diffusion operator, $\alpha$ and $\beta$ are two parameters. The iterations of this process are stopped when there is only very small changes of image pixels after the anisotropic filtering.

\section{Process for the Edge Pixels}

At each edge pixel, a $5 \times 5$ window with the edge pixel is first processed by the edge direction quantization. In this quantization, the gradient angles of the pixels are first quantized into the $0, \frac{\pi}{4}, \frac{\pi}{2}, \frac{3 \pi}{4}$ angles. Then, the highresolution edge pixel is at the center of this window and the 25 pixels in the window are at the low-resolution locations. After this, the cubic spline interpolation is carried out along the edge line with the quantized angle and in the $5 \times 5$ window to determine the image value of the center edge pixel. In this process, the edge pixels are only filtered along the edge directions and there is little edge blur artifact, which is the main advantage of the proposed algorithm.

\section{The Degradation Process and the Iterative Update Processes}

After all the pixels are up-scaled, a degradation process is carried for the currently reconstructed high resolution image to simulated the degradation of a high resolution image to the low resolution image. The following operator is convoluted with the high-resolution image values to obtain image values. 


$$
P=\frac{1}{65}\left[\begin{array}{lllll}
1 & 2 & 3 & 2 & 1 \\
2 & 3 & 4 & 3 & 2 \\
3 & 4 & 5 & 4 & 3 \\
2 & 3 & 4 & 3 & 2 \\
1 & 2 & 3 & 2 & 1
\end{array}\right]
$$

Then, the sub-sampling process is carried out to select the low-resolution pixels. Then, the pixels whose difference value between the original image value and the degraded image value are larger than a threshold are selected. The pixels around these pixels needs to be updated to get better image quality and this is the one reason that the proposed algorithm has a better performance than the previous interpolation algorithms. In these algorithm, they do not employ this iterative update process. The image values of the high resolution pixel which is around a selected pixel are recomputed using the following differential anisotropic operator.

$$
f(i, j, t+1)=f(i, j, t)+\sum_{m=1}^{4} c_{m}\left(g_{m}-g_{m}^{\prime}\right)
$$

Where $g_{m}$ and $g_{m}^{\prime}$ are the original low-resolution image values and the degraded low-resolution image values, respectively, and they are within the four nearest neighbors to the current high-resolution image pixel to be updated, and $c_{m}$ is the m-th coefficient which is inversely proportional to the distance between the m-th lowresolution pixel location and the current high-resolution pixel to be updated. $f(i, j, t)$ is the high-resolution image value at the pixel with the location of $(i, j)$ and the update iteration of $t$.

When all the pixels around the selected pixel are processed, another iteration of the degradation and recomputed process is carried out until there are none selected pixels in the simulated low-resolution image. At this point, the reconstructed high-resolution image is the result of the proposed algorithm.

\section{E. Advantages of the Proposed Algorithm}

From the above descriptions, it can be seen that the proposed algorithm has the following advantages. (1) The image edge pixels are processed separately to reduce the image blur. (2) The update process can further boost the system performance.

\section{EXPERIMENTAL RESULTS}

In the experiments of the proposed algorithm, the parameters of the algorithm are optimally set as follows. Through a large number of experiments, the suitable value for $T_{1}$ is 100 . the pixel whose $G_{m}>T_{1}$ is set as the edge pixel. In equation 7, the parameters of $\alpha$ and $\beta$ are set as 0.1 and 2 , respectively. The function of $m(x)$ is set as Hamming window function in the interval of $[0,100]$.

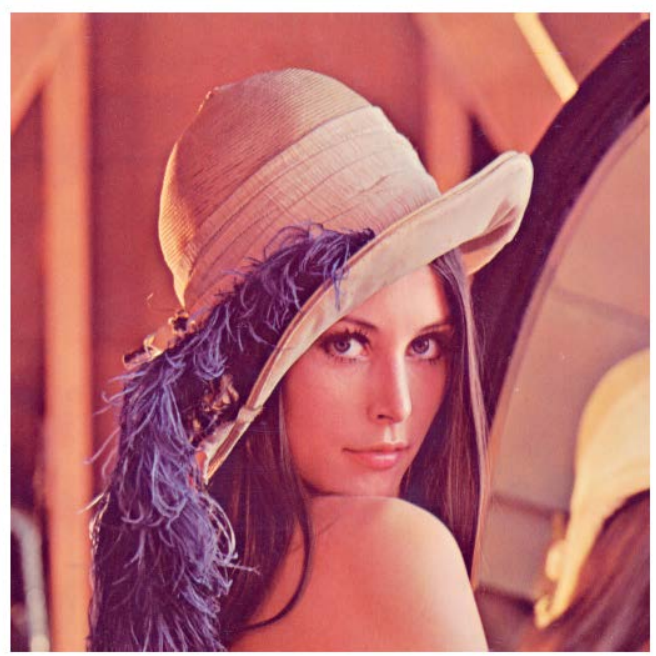

Figure 1. The original Lena image.

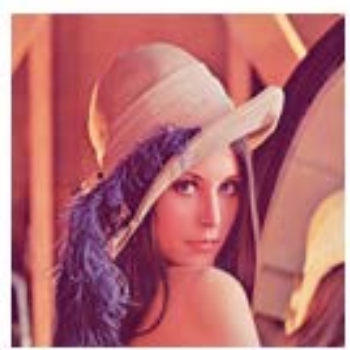

Figure 2. The degraded Lena image.

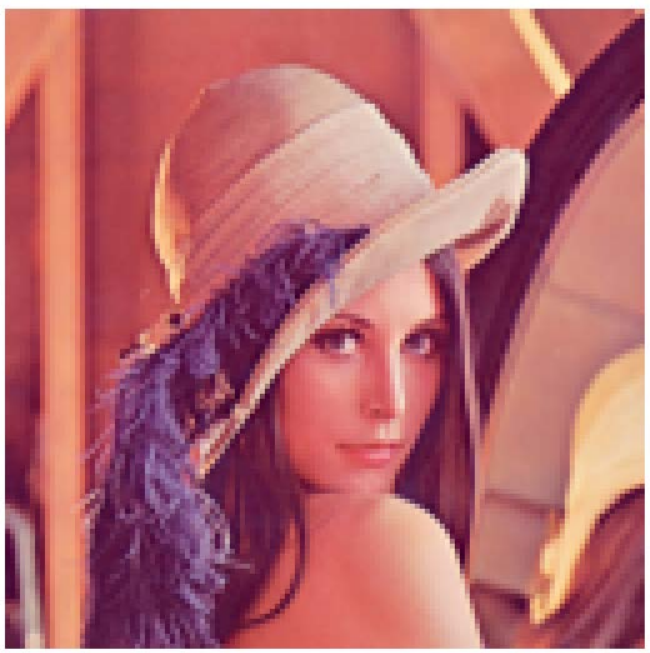

Figure 3. The super-resolution image using the bi-cubic interpolation method.

The test images are selected from the website of the signal and image processing institute of the University of 
Southern California. The super-resolution with the upscaled factor of $2 \times 2$ is tested, i.e. the test images are firstly convoluted with the operator expressed in the equation 8 , and then are sub-sampled by choosing every fourth pixels horizontally and vertically. The down-sampled images are then treated as the original images to be magnified in the proposed super-resolution algorithm. Then, the proposed algorithm is utilized to super-resolution these images with the factor of $2 \times 2$. The quality of various super-resolution algorithms is measured using the original test images and the images reconstructed by the superresolution algorithm. The measurement of the peak signal to noise ratio (PSNR) is utilized to evaluate the performance of the various algorithm. Since the bi-cubic interpolation algorithm is the currently used algorithm and has a relatively good performance, the proposed algorithm is compared with this algorithm.

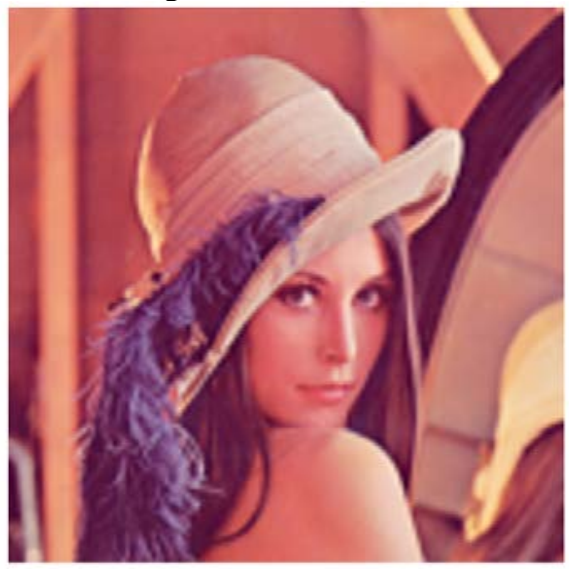

Figure 4. The super-resolution result using the proposed algorithm

In the above figures, figure 1 is the original image of Lena, and figure 2 is the degraded image of Lena with the sub-sampling factor of $2 \times 2$. Figure 3 and 4 are the superresolution results of the bi-cubic interpolation algorithm and the proposed algorithm, respectively. From these images, it can be seen that the quality of the reconstructed images of the proposed algorithm is better than that of the bi-cubic interpolation algorithm.

TABLE 1. THE PSNR COMPARISON FOR THE ALGORITHMS

\begin{tabular}{|c|c|c|}
\hline Images & $\begin{array}{c}\text { Bi-cubic } \\
\text { interpolation }\end{array}$ & $\begin{array}{c}\text { Proposed } \\
\text { algorithm }\end{array}$ \\
\hline Lena & 26.95 & 28.76 \\
\hline Baboon & 20.27 & 20.65 \\
\hline House & 24.89 & 26.55 \\
\hline Peppers & 25.68 & 27.40 \\
\hline
\end{tabular}

TABLE II.THE SSIM COMPARISON FOR THE ALGORITHMS

\begin{tabular}{|c|c|c|}
\hline Images & $\begin{array}{c}\text { Bi-cubic } \\
\text { interpolation }\end{array}$ & $\begin{array}{c}\text { Proposed } \\
\text { algorithm }\end{array}$ \\
\hline Lena & 0.72 & 0.77 \\
\hline Baboon & 0.40 & 0.43 \\
\hline House & 0.71 & 0.75 \\
\hline Peppers & 0.70 & 0.75 \\
\hline
\end{tabular}

Tables 1 and 2 lists the simulation results of the PSNR and the SSIM measures of the bi-cubic and the proposed interpolation algorithms, respectively, for various images. From these tables, it can be seen that the proposed algorithm performs better than the bi-cubic interpolation algorithm.

\section{CONCLUSIONS}

In this paper, a new interpolation based image superresolution algorithm has been proposed. The Prewitt operator is used to extract the image edge pixels. Then, the bi-linear interpolation with the anisotropic filtering is applied for the non-edge pixels, and the cubic spline interpolation along the edge direction is applied to the edge pixels, so that the shortcoming of the image edge blurs in the traditional image super-resolution algorithms can be avoided. Moreover, an iterative update process is proposed to boost the system performance, so that the degraded highresolution image is very similar to the original lowresolution image to reduce the super-resolution errors. Experimental results have been conducted for the various images, and they have demonstrated that the performance of the proposed algorithm is much better than conventional interpolation-based super-resolution algorithms.

\section{ACKNOWLEDGMENT}

This work was supported in part by the Natural Science Foundation of Zhejiang Province of China (No: LY15F010007) and the National Natural Science Foundation of China (Nos. 61571104, 61071124), and the . The authors wish to thank the reviewers for their helpful comments.

\section{REFERENCES}

[1] Aly, H.A and Dubois, E., "Image up-sampling using total-variation regularization with a new observation model," IEEE Trans. on Image Processing, vol.14, no.10, Oct 2005, pp. 1647-1659.

[2] T. Blu, P. Thévenaz, and M. Unser, "Linear interpolation revitalized," IEEE Trans. on Image Processing, vol. 13, no. 5, May 2004, pp. 710-719. [3] H. S. Hou and H. C. Andrews, "Cubic splines for image interpolation and digital filtering," IEEE Trans. on Acoust., Speech, Signal Process, vol. 26, no. 6, Dec. 1978, pp. 508-517.

[4] R. G. Keys, "Cubic convolution interpolation for digital image processing," IEEE Trans. on Acoust., Speech, Signal Process, vol. 29, no. 6, Dec. 1981, pp. 1153-1160.

[5] T. Lehmann, C. Gönner, and K. Spitzer, "Addendum: B-spline interpolation in medical image processing," IEEE Trans. on Med. Imag., vol. 20, no. 7, Jul. 2001, pp. 660-665.

[6] H. Chang, D. Y. Yeung, Y. Xiong, "Super-resolution through neighbor embedding,” Proc. 2012 IEEE Conference on Computer Vision and Pattern Recognition, IEEE press, 2012:275-282.

[7] XU Rong , JIANG Feng, YAO Hongxun, "The overview of the manifold learning,” Journal of Intelligent System, 2006 vol. 1, pp. 44-50.

[8] Zhi Jin, Tammam Tillo, Chao Yao, Jimin Xiao, Yao Zhao, "Virtualview-assisted video super-resolution and enhancement," IEEE Tran. On Circuits and Systems for Video Technology, vol. 26. no. 3, March 2016, pp. 467-478

[9] CAO Mingming, GAN Zongliang, ZHU Xiuchang, "Super-resolution algorithm through neighbor embedding with new feature selection and example training," Proc. Signal Processing (ICSP 2012), vol. 2:825-828. 